Micro-titrations involve the use of accurate volumetric apparatus, and a knowledge of the errors to be found in volumetric analysis is obviously essential for an appreciation of the limits of the accuracy obtained in such estimations. One third of Prof. Conway's book is devoted to a very complete and valuable treatment of the errors of volumetric titration, of the errors encountered in the use of pipettes and burettes and of the corrections which have to be applied for accuracy.

A chapter on the principles of colorimetry and spectrophotometry as used for micro-analytical work, and one on the micro-techniques of Kirk and of Linderström-Lang and Holter, are useful additions to the book.

This volume is of great value to all workers interested in microchemical technique. It is excellently produced and printed, with copious drawings, curves and references. Its only drawback is its relatively high price, which might put it out of the reach of many of the students for which it is intended.
J. H. QUASTEL.

\title{
PROBABILITY AND RELATIVE FREQUENCY
}

\section{Probability and Frequency}

By Prof. H. C. Plummer. Pp. xi +278 . (London: Macmillan and Co., Ltd., 1940.) 15s. net.

$\mathrm{T}$ E approach to probability adopted in this book is conventional, but cautious. The two definitions, the one a priori, based on equally Jikely aspects of a system, the second a posteriori, based on relative frequency in repeated trials, are stated and criticized, the first being preferred, but with qualifications. In the first two long chapters sixty illustrative examples of the most varied kind, divided equally between discrete and continuous problems, are posed, solved and commented upon. The reader who has worked through these will have learned a great deal about cards, dice, the Problem of Points, the St. Petersburg Paradox, Laplace's Law of Succession, Buffon's needle and many ingenious and interesting problems in geometrical probability; he should indeed be able to attack almost any reasonable problem of the kind.

In the long chapter on the Theory of Errors the normal law is derived first by Herschel's considerations, then by other sets of postulates, the conclusion being that it is "an empirical rule which is supported by experience in general, but which in the last resort must be verified by the same test". The principle of Least Squares is derived from the assumptions of normal distribution of error, and maximum probability; the other derivation, by minimum variance of consistent linear estimate, is mentioned, but as of secondary importance. (The present reviewer cannot agree with this assignment, nor with the statement at the end of $\S 168$, p. 162 , that "the basis of probability" is abandoned in this second approach ; and incidentally it is not necessary for its validity that the law of error should be symmetrical.) The treatment and notation follow classical tradition.

The statistical distributions in one variable treated in Chapter iv are those of Poisson, the
Type A Series with the cubic correction term, and Pearson's system, introduced by way of the limiting case of the difference equation of the hypergeometric distribution. The point of view of curve-fitting is empirical. "The results have only to be justified by success."

The criterion of "success", Pearson's $\chi^{2}$, nominally appears in Chapter $\mathrm{v}$, devoted to correlation, including the normal correlation function in two and three variables, correlation ratio and contingency; but also containing derivations of the distributions of $\chi^{2}$, of the estimate of variance, of Student's ratio and of the ratio of two mean squares, and finally of R. A. Fisher's distribution of $r$, with the hyperbolic tangent trans. formation to approximate normality. The inclusion of these distributions adds very considerably to the value of the book. Tables of $\log _{10} \Gamma(x)$ and of the normal probability integral (in the form in which the modulus of precision is the parameter) are appended.

The book is a welcome addition to the rather exiguous list of text-books in English on the subject. Lack of space is doubtless the cause of the omission of certain important topics, such as the theory of estimation of parameters in a probability function. In a few places more precise statements could have been made. For example (p. 250), in the matter of the $\chi^{2}$ test with reduced degrees of freedom, it should be stressed that the relations used in fitting the curve should be linear in the class-frequency deviations. Again (p. 226), for $r$ to be zero it is not necessary that one or other of the distributions of $x$ and $y$ should be symmetrical; their independence is sufficient. And lastly the reviewer, holding as he does that to keep an open mind is to assign no probability whatever, must demur to the suggestion (p. 3) that "ignorance may be an element conducing to equal likeliness". These, however, are mere obiter dicta ; the book is at all times interesting, and punctuated with dry common sense. A. C. AinkEN. 